\title{
Focused ion beam iodine-enhanced etching of high aspect ratio holes in InP photonic crystals
}

\author{
V. Callegari ${ }^{\mathrm{a})}$ \\ Swiss Federal Laboratories for Materials Testing and Research, EMPA,CH-8600 Duebendorf, Switzerland \\ and Swiss Federal Institute of Technology, ETHZ,CH-8092 Zurich, Switzerland \\ P. M. Nellen and J. Kaufmann \\ Swiss Federal Laboratories for Materials Testing and Research, EMPA,CH-8600 Duebendorf, Switzerland \\ P. Strasser and F. Robin \\ Swiss Federal Institute of Technology, ETHZ,CH-8092 Zurich, Switzerland \\ U. Sennhauser \\ Swiss Federal Laboratories for Materials Testing and Research, EMPA,CH-8600 Duebendorf, Switzerland
}

(Received 10 June 2007; accepted 8 October 2007; published 7 December 2007)

\begin{abstract}
The authors investigate the focused ion beam (FIB) fabrication of photonic crystal ( $\mathrm{PhC}$ ) holes in InP combining physical sputtering using $30 \mathrm{keV}$ gallium ions and iodine-enhanced etching at $150{ }^{\circ} \mathrm{C}$ with a protective platinum or tungsten layer. The authors show that it is equivalent to conventional dry-etching methods such as inductively coupled plasma reactive ion etching in terms of achievable aspect ratio $(>10)$, surface roughness $(12.6 \mathrm{~nm} \mathrm{rms})$, and stoichiometry. The protective layers can be selectively removed after fabrication. This new FIB fabrication method lifts the previous constraints of funnel-shaped holes and limited aspect ratios of physical sputtering and reduces the Ga implantation and amorphous layer. Thus, rapid prototyping ( $5 \mathrm{~s}$ fabrication time per hole) of planar PhCs or planar PhC elements, such as PhC $Y$ splitters, becomes feasible. (C) 2007 American Vacuum Society. [DOI: 10.1116/1.2804607]
\end{abstract}

\section{INTRODUCTION}

Photonic crystals $(\mathrm{PhCs})$ are metamaterials with a periodic spatial variation of the index of refraction. They allow for tailoring the dispersion relation of light and may exhibit forbidden frequency bands for photons, called photonic band gaps, within which no light propagation is allowed. ${ }^{1}$ Planar PhCs generally consist of air-filled holes in a dielectric background substrate, thus influencing the light propagation in the horizontal plane perpendicular to the PhC. In the third dimension, the light is typically confined by a slab waveguide structure.

Indium phosphide (InP) is a direct-band gap semiconductor suitable for fabrication of active photonic devices such as semiconductor lasers at the $1.55 \mu \mathrm{m}$ telecommunication wavelength. We work on PhCs in this material system, using air holes in the horizontal plane and an InP/InGaAsP layer stack with respective thicknesses $300 \mathrm{~nm} / 522 \mathrm{~nm}$ grown by metalorganic chemical vapor epitaxy on an InP substrate in the vertical direction to guide the light. The lattice-matched InGaAsP quaternary material has a low refractive index contrast of $\Delta n=0.18$ to InP. Due to the low index contrast, the vertical optical mode is not tightly confined in the waveguide layer, but extends several microns $(\mu \mathrm{m})$ deep into the InP substrate. For applications at $\lambda=1.55 \mu \mathrm{m}$, typical hole diameters and lattice constants are about 250 and $400 \mathrm{~nm}$, respectively. Thus, aspect ratios exceeding 10 have to be achieved with sidewall angles close to $90^{\circ}$ to keep out-ofplane scattering low.

${ }^{a)}$ Electronic mail: victor.callegari@empa.ch
Dry etching techniques, such as inductively coupled plasma reactive ion etching (ICP-RIE), have been used successfully to etch high aspect ratio structures. ${ }^{2}$ In this article, we show that focused ion beam (FIB) milling, assisted by molecular iodine, is capable to achieve structures in InP/ InGaAsP/InP with similar aspect ratio and fabrication quality than ICP-RIE. Considering that FIB etching is a maskless process, rapid prototyping and testing of $\mathrm{PhC}$ structures becomes feasible.

\section{EXPERIMENTAL PROCEDURES}

A dual-beam FIB instrument ( $30 \mathrm{keV} \mathrm{Ga}{ }^{+}$ions) was used for all etching experiments. Molecular iodine, stored in a heated crucible, was inserted into the vacuum chamber with a base pressure of $2 \times 10^{-6}$ mbar through nozzles positioned $\sim 100 \mu \mathrm{m}$ above the InP surface. The crucible was heated to $35{ }^{\circ} \mathrm{C}$ for the needle used for the etch-rate measurements and to $30{ }^{\circ} \mathrm{C}$ for the coaxial needle, which directs more gas molecules into the holes, used for high-aspect ratio structures. The latter needle has a circular opening of $300 \mu \mathrm{m}$ diameter, through which the ion beam is directed. For both needles, the gas flux on the surface strongly depends on the nozzle-substrate distance. A heating stage was used to heat the InP substrate in situ up to $350{ }^{\circ} \mathrm{C}$. The temperature was measured on the chromium-coated copper surface of the heating stage using a $\mathrm{NiCr}-\mathrm{Ni}$ thermocouple. The characterization of the hole depths and thicknesses of the deposited layers is performed with scanning electron microscopy (SEM) on FIB cross sections, whereas the hole diameters were determined by scanning ion microscopy (SIM). Sputter 


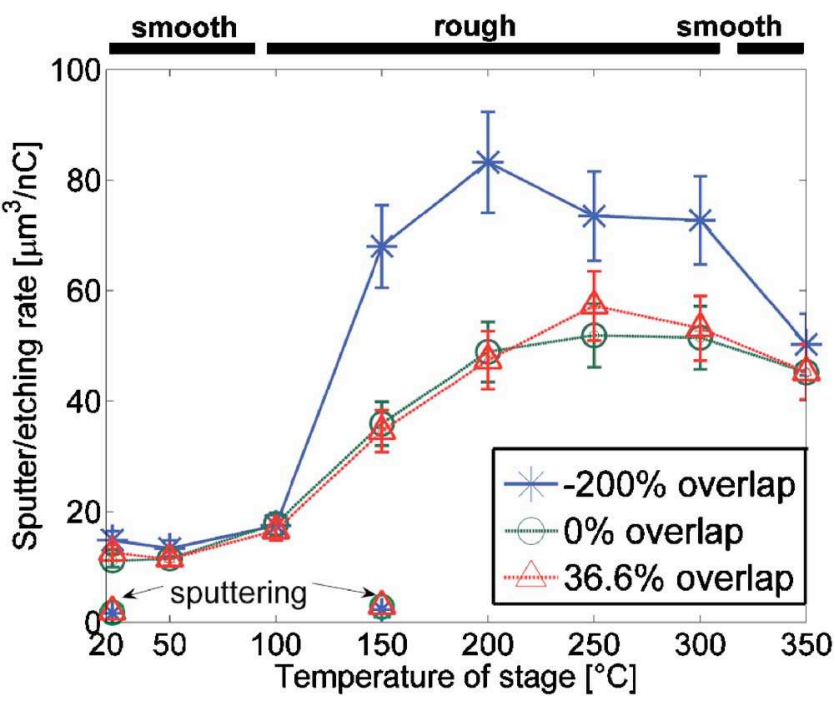

FIG. 1. Sputtering and etching rates determined by SEM measurements of FIB-sputtered cross sections as a function of stage temperature and beam overlap (InGaAsP is not shown). Surface roughness was inspected by SEM micrographs. Maximum etch rate occurs at $200{ }^{\circ} \mathrm{C}$, but with poor surface quality. Best fabrication conditions are at $150{ }^{\circ} \mathrm{C}$ and $-200 \%$ overlap. The standard deviation has been determined by measuring 18 identical squares and is $12 \%$.

and etch rates were determined by irradiating $20 \mu \mathrm{m}$ wide squares with an ion current of $313 \mathrm{pA}$ and dwell time of 100 ns during $108 \mathrm{~s}$ in InP with and without $\mathrm{I}_{2}$ and measuring the depth of the holes by SEM. The beam was scanned in a raster pattern to allow gas replenishment on the surface between irradiations. Beam overlaps $\mathrm{Ol}=(D-P) / D$, where $\mathrm{Ol}$ is the overlap, $P$ the distance between irradiated pixels, and $D$ the beam diameter, were calculated for beam diameters $D$ of $25 \mathrm{~nm}$ at $300 \mathrm{pA}$ and $10 \mathrm{~nm}$ at $10 \mathrm{pA}$. Atomic force microscopy (contact mode in air, $\mathrm{SiN}_{x}$ tip) was used to determine the surface roughness at the bottom of sputtered or etched squares. X-ray photoelectron spectroscopy (XPS) (Al $K \alpha$ line, base pressure $<10^{-9}$ mbar) was utilized to analyze the surface stoichiometry and the Ga content. The depth profile was acquired by alternating measurement of the photoelectron energies and sputtering of the area of interest with 3 $\mathrm{keV} \mathrm{Ar}^{+}$at $45^{\circ}$ angle of incidence. The atomic concentrations were calculated with Shirley background subtraction and normalization to $100 \%$. The $Y$ splitters were characterized with an end-fire setup, ${ }^{3}$ using two tunable laser sources with a wavelength range from 1470 to $1630 \mathrm{~nm}$.

\section{RESULTS AND DISCUSSION}

\section{A. Milling rate}

The sputter rates of InP and InGaAsP were determined at 1.27 and $0.36 \mu \mathrm{m}^{3} / \mathrm{nC}$, respectively. Both do not vary with temperature (Fig. 1). The sputter rate of InP is accordingly about nine times larger than the one of $\mathrm{Si}$. Therefore InP allows faster fabrication times, but is more sensitive to ion irradiation. In the case of sputtering, the depth does not vary considerably with the beam overlap, as for low aspect ratio structures $(20 \times 20 \times 0.15 \mu \mathrm{m})$, redeposition is not impor- tant (the depth of a sputtered crater at a single pixel at $0^{\circ}$ angle of incidence is $<1 \mathrm{~nm}$ for InP, allowing sputtered particles to escape efficiently). Therefore, the depth is directly proportional to the ion fluence, which was kept constant by adapting the number of passes for each beam overlap. When adding $\mathrm{I}_{2}$ to the sputter process at room temperature, we observe about a tenfold increase of the etching rate (Fig. 1), due to the spontaneous chemical reaction of $\mathrm{I}_{2}$ with nonoxidized InP and $\mathrm{Ga}$ and ion-induced intermixing, namely an ion-enhanced diffusion of $\mathrm{I}_{2}$ from the surface to the bulk and from reaction products from the underlying bulk to the surface, and the creation of numerous dangling bonds. ${ }^{4}$ The ioninduced intermixing has a range of $62 \pm 6 \mathrm{~nm} .^{5}$ However, $\mathrm{InI}_{3}$ does not sublimate but remains on the surface, which limits the etching rate. ${ }^{6} \mathrm{InI}_{3}$ starts to desorb above $80^{\circ} \mathrm{C}$ (Ref. 7) and we observe a maximum etching rate of $83.2 \mu \mathrm{m}^{3} / \mathrm{nC}$ at $200{ }^{\circ} \mathrm{C}$ stage temperature, a 66 -fold increase over the sputter rate. This increase can be explained by the thermal desorption of $\mathrm{InI}_{3}$ from the surface. At temperatures above $200{ }^{\circ} \mathrm{C}$, the etching rate decreases (Fig. 1). This is due to the lower sticking probability of $\mathrm{I}_{2}$ on the hot surface or the thermal desorption of $\mathrm{I}_{2}$ before a chemical reaction can occur. Above $330{ }^{\circ} \mathrm{C}, \mathrm{InI}_{3}$ decomposes into involatile $\mathrm{InI}$ and $\operatorname{InI}_{2}$ and volatile $\mathrm{I}_{2}{ }^{8}{ }^{8} \operatorname{InI}_{x}(x=1,2)$ forms a passivating layer which leads to a decreased etching rate at $350{ }^{\circ} \mathrm{C}$ because of the lower chemical contribution. At even higher temperatures, $\mathrm{P}_{2}$ evaporates noncongruently. ${ }^{9}$ From Fig. 1 it can be clearly seen that the beam overlap influences the etching rate, especially between $-200 \%$ and $0 \%$. By increasing the overlap, the dwell time, or the current, the ion fluence increases and more Ga will impinge per pixel, resulting in faster transition to the sputtering regime due to local $\mathrm{I}_{2}$ depletion and thus lower etching rate. In order to minimize fabrication times while maintaining a good surface quality, we chose a beam overlap of $-200 \%, 100 \mathrm{~ns}$ dwell time, and a stage temperature of $150{ }^{\circ} \mathrm{C}$ to etch the holes. At this temperature, the etching rate of $\mathrm{InP}$ is 51 times larger than the sputter rate.

\section{B. Surface roughness}

Surface roughness was determined by etching $50 \mu \mathrm{m}$ broad squares at different stage temperatures with a fluence of $2.3 \times 10^{15} \mathrm{ions} / \mathrm{cm}^{2}$. We found that surface roughness increases with temperature up to $300{ }^{\circ} \mathrm{C}$ (Fig. 1). This may be due to the spontaneous formation and desorption of $\mathrm{InI}_{3}, \mathrm{PI}_{3}$, $\mathrm{P}_{2}$, and $\mathrm{GaI}_{3}$, aided by ion intermixing. By raising the stage temperature, a more efficient thermal desorption of the reaction products occurs, which results in craters and a larger surface roughness. Since the chemical etching rate is much larger than the physical sputtering rate, the surface roughness is no longer dictated by the beam overlap and does not vary considerably with the latter. Above $300{ }^{\circ} \mathrm{C}$, the passivating $\operatorname{InI}_{x}(x=1,2)$ layer prevents strong chemical etching and the surface becomes smoother accordingly. At $150{ }^{\circ} \mathrm{C}$, we determined the surface roughness to be $12.6 \mathrm{~nm}$ rms at the bottom of the $250 \mathrm{~nm}$ deep square over an area of $25 \mu \mathrm{m}^{2}$ [Fig. 2(a)]. We achieve similar rms surface roughness as was re- 


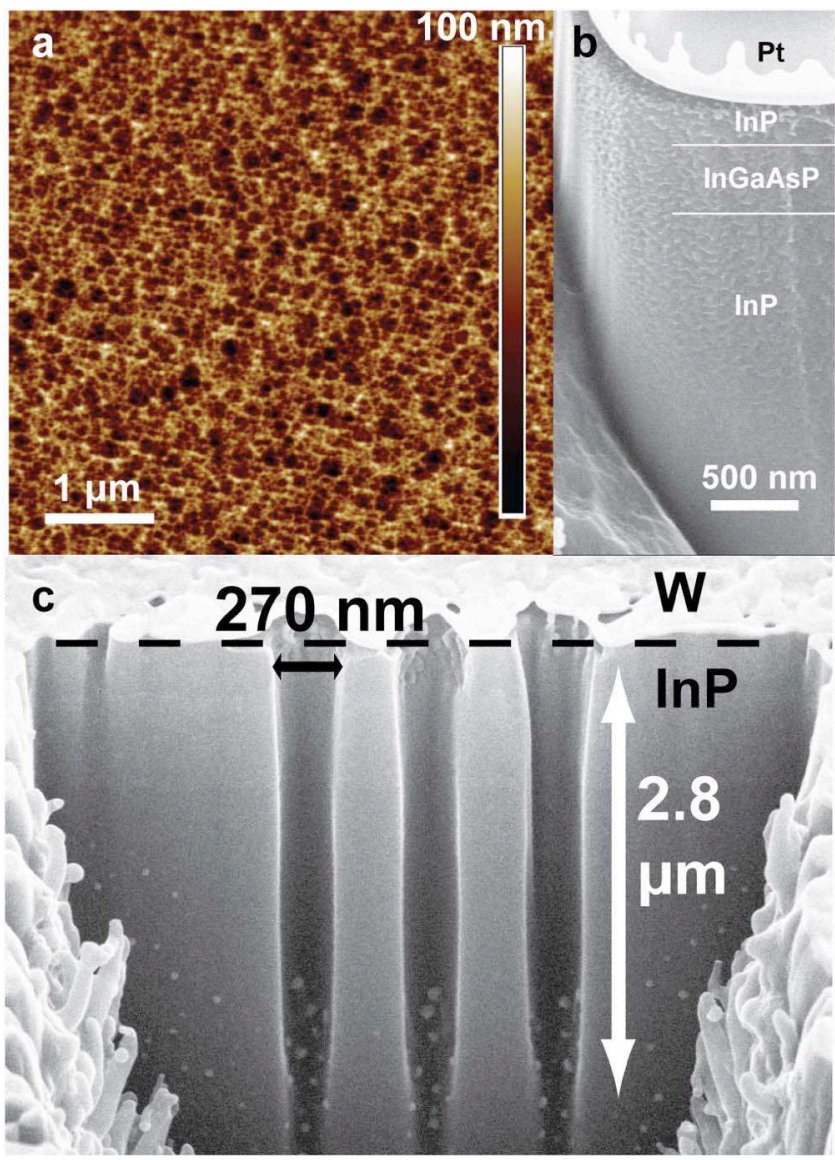

FIG. 2. (a) Atomic force microscopy measurement of the InP surface etched at $150{ }^{\circ} \mathrm{C}$. rms roughness is $12.6 \mathrm{~nm}$ over $25 \mu \mathrm{m}^{2}$. (b) SEM micrograph of a facet etched at $150{ }^{\circ} \mathrm{C}$ in InP/InGaAsP/InP. No differential etching between $\mathrm{InP}$ and $\mathrm{InGaAsP}$ is observed. Pt was deposited to protect the InP surface. (c) SEM micrograph of holes etched at $150{ }^{\circ} \mathrm{C}$ in two stages: first $3 \mathrm{~s}$ per hole at $10 \mathrm{pA}$ with a beam overlap of $36.6 \%$ and dwell time of $52.1 \mu \mathrm{s}$ to sputter through the $113 \mathrm{~nm}$ thick W mask. Subsequently, $\mathrm{I}_{2}$ is added and the beam overlap changed to $0 \%$ with a dwell time of $100 \mathrm{~ns}$ during $2 \mathrm{~s}$. The maximum aspect ratio achieved is larger than 10 .

ported for RIE. ${ }^{10}$ rms surface roughness for iodine-assisted etching at room temperature was determined to be $2.7 \mathrm{~nm}$, due to the presence of $\mathrm{InI}_{3}$ islands. Physical sputtering of $\mathrm{InP}$ has a rms surface roughness of $1.3 \mathrm{~nm}$, due to the presence of In islands. ${ }^{6}$ Here we summarize the measured rms surface roughnesses:

1. unirradiated InP wafer: $0.3 \mathrm{~nm}$,

2. sputtering at RT: $1.3 \mathrm{~nm}$,

3. iodine-assisted etching at RT: $2.7 \mathrm{~nm}$, and

4. iodine-assisted etching at $150{ }^{\circ} \mathrm{C}: 12.6 \mathrm{~nm}$.

For $\mathrm{PhC}$ holes, however, the sidewall roughness is more important than the roughness at the bottom of the hole. To estimate the roughness, we simulated the longitudinal range and straggling (standard deviation) of $30 \mathrm{keV} \mathrm{Ga}^{+}$ions impinging on InP with SRIM (Ref. 11) for incidence angles of $0^{\circ}$ and $85^{\circ}$, corresponding to incidence at the bottom of the hole, and on a sidewall, respectively. The calculations showed that the longitudinal range and straggling of $\mathrm{Ga}^{+}$ions at the bottom of the hole is $21 \pm 11 \mathrm{~nm}$. Under grazing inci-

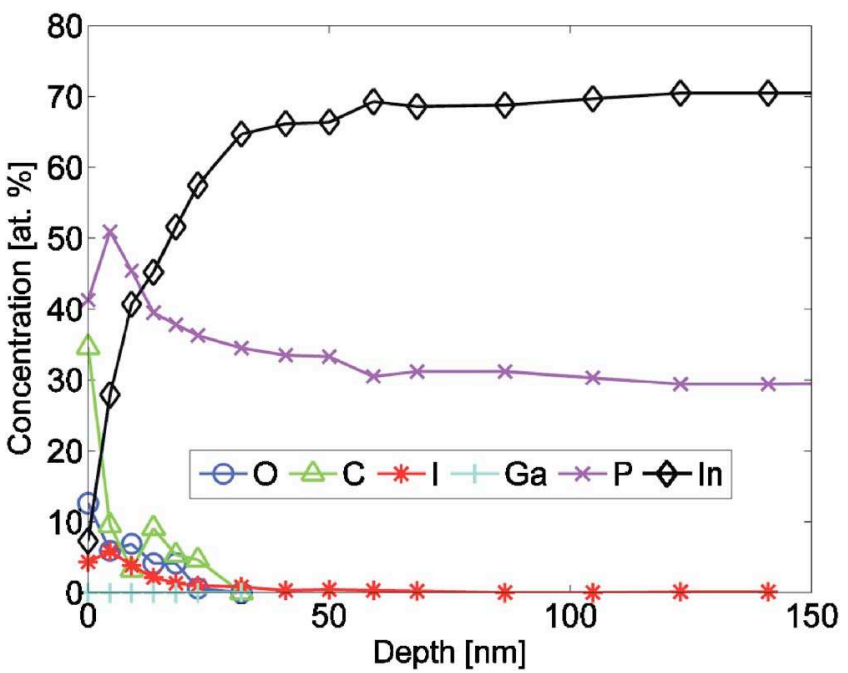

FIG. 3. XPS depth profile of the bottom area of a square etched at $150{ }^{\circ} \mathrm{C}$ with a beam overlap of $-200 \%$. The surface is $\mathrm{P}$ rich and contains $\mathrm{O}$ and $\mathrm{C}$ resulting from the transfer in air from the FIB to the XPS system. Iodine contamination is low and Ga contamination is below the detection threshold. Steady-state conditions are reached after a depth of about $70 \mathrm{~nm}$. The In-rich steady-state is a result of preferential sputtering of $\mathrm{P}_{2}$.

dence it decreases to $10 \pm 7 \mathrm{~nm}$. Accordingly, we expect the surface roughness to be smaller on the sidewalls than on the etched surface perpendicular to the ion beam.

\section{Ga implantation and stoichiometry}

$\mathrm{Ga}$ irradiation leads to $\mathrm{Ga}$ implantation and results in damage to the crystal lattice by the creation of interstitials and vacancies and ultimately amorphization of the monocrystalline InP substrate for fluences above $1.7 \times 10^{13} \mathrm{~cm}^{-2}$ (Ref. 12) which will scatter light. Ga implantation also changes the index of refraction. Both factors have an influence on the performance of the $\mathrm{PhC}$ device and should therefore be minimized. Also the stoichiometry of InP should not be altered during fabrication, since it will induce a change of the index of refraction. The XPS measurement in Fig. 3 shows that a surface layer of $20 \mathrm{~nm}$ thickness is $\mathrm{P}$ enriched. This is explained by a favored reaction of $I_{2}$ with In. For depths larger than $70 \mathrm{~nm}$, the In and $\mathrm{P}$ concentrations are constant and In rich, a result of the preferential removal of $\mathrm{P}_{2}$ from $\mathrm{InP}$ due to the physical sputtering by $\mathrm{Ar}^{+}$to acquire the depth profile. ${ }^{13}$ Iodine is detected up to a depth of $\sim 50 \mathrm{~nm}$. This can be attributed to trapped $\operatorname{InI}_{x}(x=1-3)$ or remaining $\operatorname{InI}_{x}(x=1-2)$ reaction products on the surface, which sublimate only at higher temperatures. Ga atomic concentration is below detection threshold at all depths, whereas the Ga content in InP after sputtering exceeds 20 at. \% at a depth of 20 $\mathrm{nm}{ }^{6}$ This is due to the greatly enhanced etching rate and the spontaneous thermal desorption of $\mathrm{GaI}_{3}$. Assuming that the range and straggling of $\mathrm{Ga}$ ions in $\mathrm{InP}$ is not altered by the addition of $\mathrm{I}_{2}$, the enhanced etching rate removes most of the Ga-contaminated surface layer and consequently, the amorphous layer is thinner. 


\section{Maximum aspect ratio}

Prior to etching holes, the InP cladding layer has to be protected from stray ion (ions outside the main Gaussian distribution, forming the "beam skirts") irradiation. To do this and prevent the formation of a funnel in InP, a tungsten $(\mathrm{W})$ or platinum $(\mathrm{Pt})$ protective layer was deposited. $\mathrm{W}$ was sputter coated in a physical vapor deposition system with a base pressure of $5 \times 10^{-8}$ mbar. Pt was deposited using electron beam chemical vapor deposition of the metalorganic precursor $\mathrm{C}_{9} \mathrm{H}_{12} \mathrm{Pt}$. Pt deposition has to be performed using the electron beam to avoid damaging the cladding layer. Considering that Pt can only be deposited at room temperature due to the decreased sticking probability and decomposition of the precursor above $40{ }^{\circ} \mathrm{C}$ and $\mathrm{W}$ has a lower sputter rate, we preferred $\mathrm{W}$ deposition. Each hole was sputtered in two steps: first, we sputtered the mask during $3 \mathrm{~s}$ at $10 \mathrm{pA}$ with a dwell time of $52.1 \mu$ s and $36.6 \%$ overlap. This ensures a uniform ion distribution. Then iodine was added and the hole was exposed to $\mathrm{I}_{2}$ and $\mathrm{Ga}^{+}$for $2 \mathrm{~s}$. The dwell time was $100 \mathrm{~ns}$ at $10 \mathrm{pA}$ and $0 \%$ overlap. W was subsequently selectively wet etched using $\mathrm{H}_{2} \mathrm{O}_{2}(30$ vol \%) under ultrasonic at room temperature for $15 \mathrm{~min}$. Pt was removed in situ by gas-enhanced etching at $150{ }^{\circ} \mathrm{C}$ stage temperature using $\mathrm{XeF}_{2}$, which permits one to use very low $\mathrm{Ga}^{+}$fluences.

The maximum achievable aspect ratio is limited by lateral underetching of InP beneath the mask caused by $\mathrm{Ga}^{+}$scattering from the heavy $\mathrm{W}$ or $\mathrm{Pt}$ atoms at the mask edge. To suppress the underetching, one can use thinner masks to suppress multiple scattering of ions while simultaneously reducing the sputtering time and the aspect ratio in the mask or reduce the etching time. No differential etching rate was observed between InP and InGaAsP and smooth facets could be produced [Fig. 2(b)]. We found that using a $113 \mathrm{~nm}$ thick $\mathrm{W}$ mask and $2 \mathrm{~s}$ etch time yields the best results and show the holes in Fig. 2(c). Good sidewall verticality and an aspect ratio of about 11 for a hole diameter of $270 \mathrm{~nm}$ is achieved.

\section{APPLICATION: PHOTONIC CRYSTAL $Y$ SPLITTER}

We demonstrate the capability of the new fabrication process on a $\mathrm{PhC} Y$-power splitter ${ }^{3}$ in which we fabricate the central hole by FIB, whereas the surrounding $\mathrm{PhC}$ is patterned by electron beam lithography (EBL) and transferred into the semiconductor by ICP-RIE etching [Fig. 4(a)]. Details of the fabrication process can be found elsewhere. ${ }^{3}$ Precise control over the positioning of the central hole is required, as this influences the intensity distribution in the two arms of the splitter. ${ }^{3}$ We further compare fully ICP-RIE fabricated power splitters with power splitters where the central hole is fabricated by iodine-enhanced FIB etching. The filling factor of the splitters is kept constant at $r / a=0.23$ for the $\mathrm{PhC}$ holes surrounding the splitter, $r$ being the hole radius and $a$ the lattice constant of the PhC. For the central hole, $r / a=0.19$ was chosen. An $80 \mathrm{~nm}$ thick W protection layer was used. The devices were cleaved after fabrication. By using lithographic tuning ${ }^{14}$ with $a=375-500 \mathrm{~nm}$ in $25 \mathrm{~nm}$ steps, the reduced wavelength range of $u=\lambda / a=0.23-0.34$ is
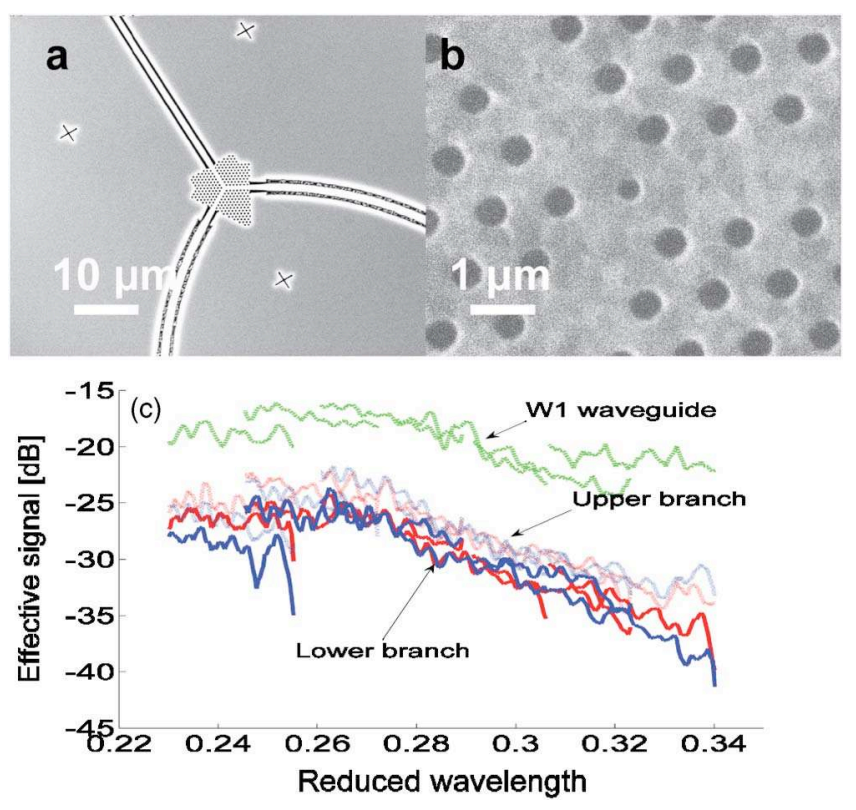

FIG. 4. (a) SEM micrograph of a power splitter fabricated by EBL/ICP-RIE without central hole. (b) SIM micrograph of the central hole fabricated by FIB in the center of the $Y$ splitter. (c) End-fire characterization of the power splitter branches (smoothed to eliminate Fabry-Pérot fringes) for EBL/ICPRIE (red) and FIB (blue) and comparison with W1 (single defect line, green) $\mathrm{PhC}$ waveguides for different lattice constants $a$.

covered. We show a power splitter with a FIB-fabricated central hole in Fig. 4(b). Figure 4(c) shows the measurement results for $W 1$ waveguides (PhCs with a single defect line) and for the two power splitter branches with FIB-fabricated and EBL/ICP-RIE fabricated central holes. Except for the tuning step with $a=375 \mathrm{~nm}$, both fabrication methods yield equivalent transmission levels. We therefore conclude that FIB fabrication quality (aspect ratio, surface roughness, and shape of the hole) is equivalent to EBL/ICP-RIE fabrication.

\section{SUMMARY}

Focused ion beam iodine-enhanced etching of InP at $150{ }^{\circ} \mathrm{C}$ is superior to physical sputtering or iodine-enhanced etching at room temperature. The etching rate at $150{ }^{\circ} \mathrm{C}$ increases 51 times over physical sputtering, enabling the reduction of the fabrication time per hole to $5 \mathrm{~s}$. The amorphous, Ga-contaminated layer formed while sputtering is greatly reduced and the buildup of $\mathrm{In}$ and $\mathrm{InI}_{3}$ islands, as occurring during sputtering and etching at room temperature, is suppressed. The surface roughness is comparable to other physical-chemical etching processes, such as ICP-RIE. However, due to the stray ions and the enhanced etching rate, the areas surrounding the fabrication area have to be protected. This can be done in situ using electron beam deposition of $\mathrm{Pt}$ and removal using $\mathrm{XeF}_{2}$-enhanced $\mathrm{FIB}$ etching. Considering the low sputter rate of $\mathrm{W}$, it is preferable to proceed with a previously sputtered $\mathrm{W}$ layer, which can be selectively wet etched by $\mathrm{H}_{2} \mathrm{O}_{2}$ after the fabrication process. Cylindrical holes with aspect ratios exceeding 10 have been obtained using this method and $\mathrm{PhC} Y$ splitters with equivalent characteristics to EBL/ICP-RIE have been fabricated and charac- 
terized. Thus, FIB rapid prototyping of InP-based planar $\mathrm{PhCs}$ becomes possible.

\section{ACKNOWLEDGMENTS}

The authors would like to thank U. Müller of EMPA for performing the XPS measurements, P. Langlet of EPFL Center of MicroNano Technology for the $\mathrm{W}$ depositions, O. Homan and E. Gini of FIRST Center of Micro- and Nanoscience of ETH Zurich for technical support and wafergrowth, respectively, as well as the financial contribution of the ETHZ INIT project "composite doped metamaterials" and EIPBN conference 2007 for the student grant.

${ }^{1}$ J. D. Joannopoulos, R. D. Meade, and J. N. Winn, Photonic CrystalsMolding the Flow of Light (Princeton University Press, Princeton, NJ, 1995).

${ }^{2}$ P. Strasser, R. Wüest, F. Robin, D. Erni, and H. Jäckel, J. Vac. Sci. Technol. B 25, 387 (2007).
${ }^{3}$ P. M. Nellen, P. Strasser, V. Callegari, R. Wüest, D. Erni, and F. Robin, Microelectron. Eng. 84, 1244 (2007).

${ }^{4}$ J. Dieleman, F. H. M. Sanders, A. W. Kolfschoten, P. C. Zalm, A. E. Devries, and A. Haring, J. Vac. Sci. Technol. B 3, 1384 (1985).

${ }^{5}$ S. Rubanov and P. R. Munroe, Microsc. Microanal. 11, 446 (2005).

${ }^{6}$ V. Callegari, P. M. Nellen, T. Yang, R. Hauert, U. Müller, F. HernándezRamírez, and U. Sennhauser, Appl. Surf. Sci. 253, 8969 (2007).

${ }^{7}$ B. Brunetti, A. Giustini, and V. Piacente, J. Chem. Thermodyn. 29, 239 (1997).

${ }^{8}$ T.N. Guliev, Izv. Vys. Ucheb. Zaved, Khim. Khim. Tekhnol. 34, 7 (1991). ${ }^{9}$ R. F. C. Farrow, J. Phys. D 7, 2436 (1974).

${ }^{10}$ J. S. Yu and Y. T. Lee, Semicond. Sci. Technol. 17, 230 (2002).

${ }^{11}$ J. F. Ziegler, J. P. Biersack, and U. Littmark, The Stopping and Range of Ions in Solids (Pergamon, New York, 1985); www.srim.org.

${ }^{12}$ R. Menzel, K. Gartner, W. Wesch, and H. Hobert, J. Appl. Phys. 88, 5658 (2000).

${ }^{13}$ W. Yu, J. L. Sullivan, S. O. Saied, and G. A. C. Jones, Nucl. Instrum. Methods Phys. Res. B 135, 250 (1998).

${ }^{14}$ R. Ferrini, D. Leuenberger, M. Mulot, M. Qiu, J. Moosburger, M. A. Kamp, A. Forchel, S. Anand, and R. Houdré, IEEE J. Quantum Electron. 38, 786 (2002). 